\title{
Subclinical Onychomycosis: Not Present in HIV-Positive Population?
}

\author{
Claudia Contreras-Rivera ${ }^{a}$ Sonia Toussaint-Caire ${ }^{b}$ Ramón Fernández-Martínez ${ }^{c}$ \\ Gabriela Moreno-Coutiño ${ }^{d}$ \\ ${ }^{a}$ Mycology Department, General Hospital Dr. Manuel Gea González, Mexico City, Mexico; bermatology Service, \\ General Hospital Dr. Manuel Gea González, Mexico City, Mexico; 'Mycology Department, General Hospital \\ Dr. Manuel Gea González, Mexico City, Mexico; d Mycology Department, General Hospital Dr. Manuel Gea González, \\ Mexico City, Mexico
}

\section{Keywords}

Subclinical onychomycosis · HIV-positive patients · Nail disease

\begin{abstract}
Background: Onychomycosis is one of the most common nail diseases and constitutes up to $50 \%$ of all nail conditions. It is a chronic fungal nail infection common in the HIV-infected population. Few studies have found fungal organisms in the nail without clinical evidence, and thus, termed subclinical onychomycosis. Objectives: We analyzed the nails of the HIV population in our hospital, searching for subclinical onychomycosis. Method: A distal nail clipping was stained with PAS and observed by a trained dermatopathologist. Results: All of our samples $(n=48)$ turned out to be negative for fungal structures. Conclusions: The epidemiology of onychomycosis is changing, treatment options are much better now, and diagnosis and infection detection are being done earlier. Therefore, we will continue to further study this disease in other patient populations so that we can compare our results and see whether the incidence of onychomycosis in the HIV population is now similar to that in those without HIV infection of the same age.

(c) 2018 S. Karger AG, Basel
\end{abstract}

() 2018 S. Karger AG, Basel

E-Mail karger@karger.com

www.karger.com/sad

\section{Introduction}

Onychomycosis is one of the most common nail diseases and constitutes up to $50 \%$ of all nail conditions, present in more than $10 \%$ of the worldwide population [1]. It is a chronic fungal nail infection which tends to gradually destruct the nail plate and is considered a source of dissemination for skin dermatophytosis [2].

These fungal infections are common in the HIV-infected population [3]. Evidence supports that the prevalence of onychomycosis in HIV-positive patients ranges from 20 to $44 \%$ and may even be an early clinical marker of immunosuppression when the CD4 count is below $450 / \mu \mathrm{L}$. A study reports predisposing factors for onychomycosis in HIV-infected patients. These include: CD4 cell count $<370$, tinea pedis, and walking barefoot around pools and other recreational areas such as gymnasiums, which are mostly the same for the non-HIV population [2].

The possibility of growing a positive culture for onychomycosis increases in patients with a viral load higher than 50 copies/mL $[1,3]$, and the most frequent clinical presentation is distal and lateral subungual onychomycosis, followed by total dystrophic onychomycosis. Parallel to this, few studies have found fungal organisms invading 
Table 1. Demographics and laboratory parameters of the HIV-positive studied population

\begin{tabular}{|c|c|c|c|c|c|c|}
\hline & \multirow{2}{*}{$\begin{array}{l}\text { Patients, } \\
n(\%)\end{array}$} & \multirow{2}{*}{$\begin{array}{l}\text { Age, } \\
\text { years }\end{array}$} & \multirow{2}{*}{$\begin{array}{l}\text { cART, } \\
n(\%)\end{array}$} & \multirow{2}{*}{$\begin{array}{l}\text { CD4 T-cell count, } \\
\text { cells } / \mu \mathrm{L}\end{array}$} & \multicolumn{2}{|l|}{ Viral load } \\
\hline & & & & & $\begin{array}{l}\text { HIV RNA, } \\
\text { copies/mL }\end{array}$ & $\begin{array}{l}\text { undetectable, } \\
n(\%)\end{array}$ \\
\hline Female & $5(10.4)$ & $32-47$ & $4(80)$ & $40-447$ & - & $3(6.259)$ \\
\hline Male & $43(89.5)$ & $22-53$ & $38(88.3)$ & $170-1,190$ & $6-979$ & $19(39.59)$ \\
\hline Total & $48(100)$ & & $42(87.5)$ & & & $22(45.8)$ \\
\hline
\end{tabular}

the nail plate without any clinical manifestations, termed subclinical onychomycosis, with a reported incidence that ranges from 1.5 to $17 \%$ depending on the series, so we aimed to study its prevalence in a Mexican HIV-infected population [4].

\section{Materials and Methods}

This is an observational, descriptive, prospective, and transversal study approved by our institutional ethics committee, that included HIV-positive adult men and women attending the Dermatology Service in the Infectious Disease Clinic at the General Hospital Dr. Manuel Gea González between August 2017 and June 2018. We invited patients that attended the clinic with or without dermatological issues, but unrelated to any fungal infection, either superficial or systemic. Patients were specifically required to have a completely clear first toenail of the right foot, that did not raise any suspicion of onychomycosis or any other nail disorder. Individuals with any visible sign of abnormality in the toenails, with very short nails, or who had undergone antifungal treatment (either oral or topical) during the last 6 months were excluded. Written informed consent was obtained from all study participants prior to examination. Information obtained included age, gender, previous history of onychomycosis and treatment received (if any), date of first HIV-positive test, antiretroviral therapy, CD4 T-cell count, and HIV viral load at the time, as well as any other medical conditions. Examination of the toenails was performed by a trained dermatologist. Nail clippings were obtained from the distal border of the right foot big toenail, sent to the histopathology laboratory for periodic acid-Schiff (PAS) staining, and observed by a dermatologist and a dermatopathologist.

\section{Results}

A total of 48 patients with a normal-appearing big right toenail and with previous diagnosis of HIV infection participated in this study: 43 men (89.5\%) and 5 women (10.4\%), aged between 22 and 53 years, with a mean age of 34 years. During the interrogatory session none reported having tinea pedis or onychomycosis diagnosis and/or treatment during the last year.
Overall, we collected the CD4 T-cell count of 29 patients $(60.4 \%)$, with a range between 40 and 1,190 cells/ $\mu \mathrm{L}$; data for the remaining 19 patients $(39.5 \%)$ were not available. Regarding the viral load, it was undetectable in 22 patients (45.8\%), in a range of 6-979 HIV RNA copies/ $\mathrm{mL}$ in 11 patients $(22.9 \%)$, and in 15 patients $(31.25 \%)$ the information was not available. Forty-two patients (87.5\%) were on combination antiretroviral therapy (cART) and $6(12.5 \%)$ had not yet started antiretroviral therapy. Fourteen of the participants were first diagnosed with HIV infection the previous year (2017), and the rest were diagnosed between 1997 and 2018 .

The number of patients, age and sex distribution, and HIV laboratory parameters are displayed in Table 1 . The 48 PAS samples were analyzed by an experienced dermatopathologist, and fungal structures could not be found in any of them.

\section{Discussion/Conclusion}

Onychomycosis is one of the most common nail diseases, with prevalence rates varying from 3 to over 25\%, as the percentage increases with age, humid climate, and presence of underlying immunosuppressive disease [6]. It is often asymptomatic, although most patients experience decreased quality of life and sometimes it can cause functional impairment [5]. Clinical signs of onychomycosis include thickened, discolored, and crumbling nails that may be partially detached from the nail bed $[7,5]$. These manifestations of nail infection signs are the determining factor that prompts the clinician to perform mycological testing. However, infectious organisms could be present despite the absence of clinical signs, and in such cases, the prevalence of fungal organisms in normal-appearing nails is not well established [7], nor is the extent of time or the amount of fungal load that is required for clinical signs to appear [5] (i.e., an "incubation" period). 
It has become widely accepted that histopathologic analysis with PAS staining of nail clippings is the "gold standard" for the diagnosis of onychomycosis. It is the most sensitive test $(98.8 \%)$ compared to other methods including standard potassium hydroxide preparation $(\mathrm{KOH})(90.9 \%)$ and culture in dermatophyte test medium (57.3\%) [8], and therefore we chose this method for our study. In addition, to improve accuracy, an experienced dermatopathologist examined the samples from which we expected positive results, with numbers at least similar to those reported by Walling [5], who found an association of $7 \%$ subclinical onychomycosis in patients with tinea pedis and only $1.5 \%$ in the control group, or to the work published by Elbendary et al. [4] who found a rate of $7.5 \%$ of subclinical onychomycosis in diabetic patients, also associated with tinea pedis.

Surprisingly, we did not find any case of subclinical onychomycosis in this population with a high incidence of onychomycosis, and comparing this with the other reports, although none of them involved a HIV-positive population, the authors mention that participants had tinea pedis, while our patients did not, so we assume this as the main risk factor for subclinical onychomycosis. Also as mentioned by other authors, our patients showed no relation between the CD4 cell count and the presence of dermatophyte infection [9].

The incidence of onychomycosis has changed since the use of prophylactic antifungal therapy with fluconazole or itraconazole and the introduction of cART $[1,2]$, which may improve onychomycosis even without antifungal therapy just by restoration of the immune function [2]. Nonetheless, in published studies it remains up to four times more frequent in these individuals than in the general population [1], with a prevalence of $34.8 \%$ and most frequently found in toenails, with only $5 \%$ in fingernails and always associated with toenail involvement [10]. In addition, males are predominantly affected (68.7\%), with a male:female ratio of 2.2:1 [2]. These reports may need to be updated, as the treatment options are much better now, and the diagnosis and infection detection are being done earlier.

We will further study this disease because we think it is important to identify the subclinical infection so that patients can be detected before the nail presents clinically evident dystrophy, thus avoiding the distress of suffering this ailment and reducing dissemination among family members. In addition, hopefully the treatment will be shorter, thereby reducing costs and drug adverse reactions.

\section{Statement of Ethics}

The subjects gave written informed consent for their participation. The study was approved by the ethics committee of the General Hospital Dr. Manuel Gea González.

\section{Disclosure Statement}

The authors have no conflicts of interest to disclose.

\section{Author Contributions}

Claudia Contreras-Rivera conducted patient inclusion and kept the records. Sonia Toussaint-Caire carried out revision of the pathology samples. Ramón Fernández-Martínez was involved in the protocol design. Gabriela Moreno-Coutiño was responsible for the protocol design and writing the paper.

\section{References}

1 Chang P, Ucelo Z, del Pilar Garzaro Chávez $\mathrm{H}$. Onychomycosis and Immunodepression. Curr Fungal Infect Rep. 2017;11(4):252-7.

2 Ruíz-López P, Moreno-Coutiño G, Fernández-Martínez R, Espinoza-Hernández J, Rodríguez-Zulueta P, Reyes-Terán G. Evaluation of improvement of onychomycosis in HIV-infected patients after initiation of combined antiretroviral therapy without antifungal treatment. Mycoses. 2015 Sep;58(9):51621.

3 Moreno-Coutiño G, Arenas R, Reyes-Terán G. Improvement in onychomycosis after initiation of combined antiretroviral therapy. Int J Dermatol. 2013 Mar;52(3):311-3.
4 Elbendary A, El Tawdy A, Zaki N, Alfishawy $\mathrm{M}$, Rateb A. Subclinical onychomycosis in patients with type II diabetes. Dermatol Rep. 2015 Dec;7(3):6099.

5 Walling HW. Subclinical onychomycosis is associated with tinea pedis. Br J Dermatol. 2009 Oct;161(4):746-9.

6 Hay RJ, Baran R. Why should we care if onychomycosis is truly onychomycosis? Br J Dermatol. 2015 Feb;172(2):316-7.

7 Shemer A, Gupta AK, Farhi R, Daigle D, Amichai $\mathrm{B}$. When is onychomycosis onychomycosis? A cross-sectional study of fungi in normal-appearing nails. Br J Dermatol. 2015 Feb; 172(2):380-3.
8 Barak O, Asarch A, Horn T. PAS is optimal for diagnosing onychomycosis. J Cutan Pathol. 2010 Oct;37(10):1038-40.

9 Rodwell GE, Bayles CL, Towersey L, Aly R. The prevalence of dermatophyte infection in patients infected with human immunodeficiency virus. Int J Dermatol. 2008 Apr;47(4): 339-43.

10 Moreno-Coutiño G, Arenas R, Reyes-Terán G. Clinical presentation of onychomycosis in hiv/aids: a review of 280 mexican cases. Indian J Dermatol. 2011 Jan;56(1):120-1. 